\title{
Surgical alveolar corticotomy a process accelerating orthodontic teeth displacements
}

\author{
Interview with Jean-David SEBAOUN*, \\ conducted and tranlated by Olivier WEISSENBACH
}

\section{O. WeIsSEnBaCH. What is your orthodontic background?}

J.-D. Sebaoun. I graduated from Boston University Goldman School of Dental Medicine, Boston, MA, USA, where I obtained a Master of Science in Orthodontics and Dentofacial Orthopedics with a specialty in oral and bone biology as well as a certificate of advanced graduate studies in orthodontics. In 2007 I became a diplomate of the American Board of Orthodontics and took a faculty position as an assistant clinical professor in orthodontics for the department of Periodontology at BU where I teach accelerated orthodontic treatments. I also practice conventional orthodontics in a dental clinic in Boston.

\footnotetext{
* Jean-David Sebaoun is an assistant clinical professor in orthodontics for the department of Periodontology at Boston University and a member of William M. WILKO and Donald J. FERGUSSON's team. They pioneered a new orthodontic process quickening orthodontic teeth displacement.
}

O.W. In France, you are a pioneer of the corticotomy facilitated orthodontic treatment. How would you define this technique?

J-D. S. Alveolar corticotomy is defined as the surgical scarifying of the alveolar cortex. Our particular technique involves a full thickness flap followed by superficial cuts through the alveolar cortex barely penetrating the medullary bone in the area where rapid tooth movement with decreased resistance is desired. An apposition bone graft is placed before suturing the flaps where dental expansion is planned and/or where weak periodontal support or dehiscences are present. As a result, 4 to 7 months active treatments are routine even for severe cases and the scope of treatment is widely expended.

\section{O.W. What is the research underlying this technique?}

There have been more than 8 years of university-based research in the field of Alveolar corticotomy including multiple case reports in the literature, Thesis, clinic tables, presentations and scientific awards. 
From a basic science standpoint, we recently, published our animal research in the Journal of Periodontology2. In which the effect of the technique was for the first time demonstrated at the histological level.

From a clinical standpoint, orthodontic outcome, stability and relapse following decortication plus grafting have been compared to conventional orthodontic treatment and our finding were published in several Thesis throughout the years. Those works were consistent on the fact that clinical outcomes were similar for corticotomy and conventional treatment but were achieved about 3 time faster and resulted in more stable retention outcomes following decortication and grafting.

Recently we published the description of the surgical technique and reported some clinical cases in the literature ${ }^{3}$.

Today our researches target the effects of the procedure at the cellular and molecular levels as well as developing variation and simplification of the surgical procedure.

The conclusions of those various researches allowed us to practice evidence-based orthodontics while treating our candidate for corticotomy in our everyday practice.

\section{O.W. Can you explain the grow-} ing interest of the orthodontic community for this new concept?

J-D. S. This concept is part of a new era in orthodontics where new technologies, new mechanics and new surgical procedures are entering our everyday practice and expending the limits of classic orthodontics.

\section{O.W. Which malocclusions are able to benefit from corticotomy?}

J-D. S. Dental Class I with mild to severe crowding are ideal candidates.

Recently, we extended our technique to Full dental Class II cases with very good success.

A limited procedure can be well suited for intrusion of single teeth or segments.

Crossbites and borderline skelettal openbites respond well to the treatment.

However it is very important to keep in consideration the soft tissue aspect of the patient during treatment planning in order to prevent any detrimental effect when a non-extraction corticotomy option is choosen.

\section{O.W. Which patients are best suited to the procedure?}

J-D. S. The technique is best suited to late teens and adult patients. It is extremely important for the patients to understand the rational for an every-2-weeks activation. They must be compliant and willing to work with us to obtain the best result is the least amount of time.

The motivation and demand for a short treatment time are decisive factors. Interdisciplinary cases and preprosthetics cases that express the desire to complete their overall treatment quickly are good candidate as well.

Other than this, they must be healthy enough to undergo a periodontal procedure.

\section{O.W. How does corticotomy pro- cedure fulfill the patients' awaits?}

J-D. S. In the past years we have noticed a significant increase for the 
demand of more cosmetic solutions in orthodontics (Lingual treatment, Invisalign ${ }^{\circledR}$...). This concern is not only expressed from females, but males and adolescents are more and more concerned about this. A procedure in which the duration of treatment can be extremely reduced while similar ideal results are obtained will fulfill the goal of today's most demanding patients.

O.W. Are the biologic conditions, age, health, periodontal health, etc. of importance for successful treatment?

J-D. S. Age is important because the patient must be mature enough to be highly compliant with his treatment. On a regular basis we do not treat patients under the age of 15 .

The candidates must be healthy enough to undergo a periodontal surgery.

From a periodontal standpoint, they must be stable before starting treatment.

\section{O.W. Which counter indications are there?}

J-D. S. Presence of active periodontal disease.

Uncontrolled osteporosis or bone disease.

Long-term use of biphosphonate, anti-inflammatory, immunosupressors and steroids.

O.W. How do you explain the treatment steps to the patient?

J-D. S. During the treatment planning appointment, we explain the timing and the different steps to the patient. It is very important that day to stress the importance of a 2 weeks activation schedule to the patients, in order to maintain a mechanical stimulation on the alveolar bone and to extend the window of opportunity in which the teeth can be moved faster.

If we judge the patient unwilling to comply with this schedule, alternative treatment plans are offered.

\section{O.W. Are there some specific preparative procedures?}

J-D. S. A periodontal consultation in which the periodontal status of the patient will be evaluated is mandatory before performing the procedure.

A cleaning and eventual scaling is then performed prior to bracketting.

The surgery is done 1 to 2 weeks after placement of the appliances.

O.W. To your mind, is it better to proceed in teamwork with a trained periodontal surgeon or that the orthodontist himself performs the surgery?

J-D. S. This particular surgery absolutely needs to be performed by a trained periodontist or oral surgeon.

During the active treatment there will also be some follow up checks of the periodontal tissue by the surgeon. Usually 2 weeks post op for suture removal and 3 months post op.

O.W. Once the treatment is decided, how is it initiated? Is the surgery the first step? Is it performed in the same appointment that the orthodontics?

J-D. S. Following the othodontic and periodontal consultations, the braces are placed and the surgery in performed 1 to 2 weeks after.

This is in order to obtain an initial osteoclastic activity due to the 
mechanical Stimulation of the braces in the area in which the surgery will be done.

The day of the surgery it is possible to remove the wires in order to simplify the access for the surgeon and they are then placed back immediately after the operation. If the surgeon feels comfortable to proceed with the wires on, they are not removed.

\section{O.W. In what does the surgical procedure consist?}

J-D. S. Following anesthesia, a full thickness flap is elevated on both the buccal and lingual of the teeth that need to be moved quickly. The untreated segments will have a higher anchorage value.

Cortical incisions are then performed as a combination of circumscribing cuts around the roots and dots. The cuts go through the cortex but just barely penetrate into the medullary bone. They are superficial and NOT through and through osteotomy cuts.

The bone grafting material is then placed (0.5 to $1 \mathrm{cc}$ per tooth) where needed and the flaps are then sutured with individual GoreTex ${ }^{\circledR}$ sutures.

\section{O.W. Is the grafting material's choice decisive?}

J-D. S. The grafting material is in NO WAY related to the increase rate of tooth movement. The sole goal of the augmentation bone graft in to reduce the risk for dehiscences and fenestration in the crowded areas where expansion will be performed.

It has also been shown that an increased cortical thickness in the anterior segment might be correlated with increased stability.
O.W. Is the choice of a piezoelectric scalpel of importance in order to simplify the surgery or to perform a less invasive procedure?

J-D. S. It is our goal today to simplify both the procedure and the post op discomfort for the patient. In that regards we do believe than a piezo-electric device plays a good role in performing a less invasive procedure.

The animal researches we are doing today show that even if the procedure is significantly less invasive, the results are identical.

O.W. Why do you choose full sizes thermic wires immediately after the surgery?

J-D. S. We do not use FULL size wire immediately after the surgery but we try to engage the heavier wire possible in order to produce the maximum amount of mechanical stimulation.

It is important to note than even if the wires are heavier than in conventional orthodontics, the discomfort for the patient is not stronger because of the low resistance and density of the bone in the area where the surgery was done. You will notice that the teeth are extremely mobile during the treatment but this is a normal stage because of the increased width of the PDL post op and this is not a sign of a periodontal pathology. During the stabilization stage, the mobility will come back to steady state level usually within a few weeks.

\section{O.W. What orthodontic adjust-} ment intervals you recommend?

J-D. S. We recommend 2 weeks interval between orthodontic 
adjustments in order to maintain the mechanical stimulation of the bone so that the window of opportunity in which the RAP is present and the teeth can be moved faster does not dissipate. If multiple appointments are missed or too much time is present between visits, the osteopenia will disappear and the alveolar bone will return to its normal physiology. We then understand why it is of primary importance for the patient to understand this and to be compliant with his visit schedule.

\section{O.W. Has the classical orthodon- tic mecanotherapy to be modified when a corticotomy is performed?}

J-D. S. The mecanotherapy itself is pretty similar. However, the amount of force applied to the teeth will be greater.

Also, we have to understand the modification of anchorage values when treating the patients. The teeth that are not in area where decortication was done will have a higher anchorage value that those that are at a close proximity from the surgical site. This fact will have to be taken into consideration when applying our mechanics.

O.W. Do all cases receive full arch corticotomy? Is it possible to reserve arch sectors for anchorage?

J-D. S. NO. Localized procedures are indicated especially when simple intercanine crowding is observed and the posterior segment shows an ideal Class I or when limited treatment as single tooth intrusion is planned.

Again, the teeth that are not in area where decortication was done will have a higher anchorage value.

\section{O.W. Does the increased alveolar bone volume improve the degree of tooth movement?}

J-D. S. NO, The increase alveolar bone volume will play a significant role in stability as well as in increasing the osseous envelope around the arches specifically in areas of poor cortical thickness and dehiscences which increase the scope of treatment in a safely manner (more stable expansion ...). However, the graft is not in any way responsible of the increased rate of tooth movement.

\section{O.W. For which cases is it possi- ble to avoid an orthognatic surgery?}

J-D. S. Borderline skeletal open bites or maxillary constriction are well suited when and only when a dental correction will not be detrimental to the esthetics of the face. For example, an anterior open bite with already a gingival display at smile will not be good to be treated by extrusion of the maxillary anteriors but might be better treated by a maxillary impaction. As in any orthodontic treatment it will also be important to understand the aetiology of the problem before treating.

In other words, corticotomy does not in any way replace orthognatic surgery, but in some specific cases might be an additional tool in the hand of the orthodontics.

\section{O.W. What about stability?}

J-D. S. Our studies compared post treatment and post-retention study cast and Panorex of over 120 corticotomy cases to conventional orthodontic cases in regard to 24 criteria of the Objective Grading System of the American Board of Orthodontics. 
Our results were conclusive and showed that the outcomes are as and even more stable for some of the criteria studied following corticotomy.

The increase stability could be correlated to the increased alveolar bone volume induced by the graft.

\section{O.W. Finally, which are the utter- most advantages of the corticoto- my procedure?}

J-D. S. Optimal orthodontic outcome achieved in shorter treatment time.

Greater alveolar bone volume

Increased scope of treatment

Very good stability.

O.W. As a conclusion of the interview, which advice could you give to those who never used the technique and who project to practice?

J-D. S. First of all, be a good treatment planner. Not all cases are good candidate for this procedure. Also I always try to place the patient as the centerpiece of the problem. We have to listen to their demand, their goal and offer them an option that would be best suited for them.

With a good diagnosis and a good understanding of the biologic rationale behind the technique, corticotomy is an amazing tool in the hand of today's clinicians.

O.W. Jean-David Sebaoun we highly appreciate the excellence of your cordial welcome. The editorial board is very grateful to your kind acceptance of this precious interview.

\section{REFERENCES}

1. Sebaoun JD, Ferguson DJ, Wilcko MT, Wilcko WM. Corticotomie alvéolaire et traitements orthodontiques rapides. Orthod Fr 2007;78(3):217-25.

2. Sebaoun JD, Kantarci A, Turner JW, Carvalho RS, Van Dyke TE, Ferguson DJ. Modeling of trabecular bone and lamina dura following selective alveolar decortication in rats. J Periodontol 2008;79(9):1679-88.

3. Sebaoun JD, Surmenian J, Ferguson DJ, Dibart S. Acceleration of Orthodontic Tooth Movement Following Selective Alveolar Decortication: Biological Rational and Outcome of an Innovative Tissue Engineering Technique. International Orthodontics 2008; 6(3):235-49. 